\title{
Programmable Quantum Interference in Complex Optical Networks Realized in Opaque Scattering Media
}

\author{
Ravitej Uppu ${ }^{1}$, Tom A. W. Wolterink ${ }^{1,2}$, Georgios Ctistis ${ }^{1,3}$, Willem L. Vos ${ }^{1}$, \\ Klaus-J. Boller ${ }^{2}$, and Pepijn W. H. Pinkse ${ }^{1}$ \\ ${ }^{1}$ Complex Photonic Systems (COPS), MESA+, Institute for Nanotechnology \\ University of Twente, P. O. Box 217, 7500 AE Enschede, The Netherlands \\ ${ }^{2}$ Laser Physics and Nonlinear Optics (LPNO), MESA+, Institute for Nanotechnology \\ University of Twente, P. O. Box 217, 7500 AE Enschede, The Netherlands \\ ${ }^{3}$ NanoBioInterface, Saxion University of Applied Sciences \\ P. O. Box 70000, 7500 KB Enschede, The Netherlands
}

\begin{abstract}
Light transport in opaque scattering media mixes the light across the large number of modes in the system. The mixing results in the scrambling of information encoded on the incident light, which is generally detrimental. However, the multimodal transport hints at the possibility of utilizing them as complex optical networks if the transport could be controlled. In recent years, wavefront shaping through adaptive phase control has been used to create multiport optical devices in opaque scattering media [1].

We study the programmability of one such device created in opaque scattering medium, a twoport beam splitter which is a primitive for any complex linear optical network. Here, I will discuss our latest results on the quantum interference between single photons from an ultrabright quantum source at the two-port beam splitter. The novel feature of the realized beam splitter is the programmability of quantum interference between single photons, creating either bunched or anti-bunched light at the outputs [2]. Our demonstration is a first step towards realizing complex optical networks with programmable quantum correlations using opaque scattering media.
\end{abstract}

\section{REFERENCES}

1. Huisman, S. R., T. J. Huisman, T. A. W. Wolterink, A. P. Mosk, and P. W. H. Pinkse, Optics Express, Vol. 23, 3102-3116, 2015.

2. Wolterink, T. A. W., R. Uppu, G. Ctistis, W. L. Vos, K.-J. Boller, and P. W. H. Pinkse, Physical Review A, to be published. 